\title{
INDIRECT BOUNDARY EXACT CONTROLLABILITY OF A MULTIDIMENSIONAL SYSTEM OF COUPLED WAVE EQUATIONS IN A REGULAR DOMAIN
}

\author{
CHEIKH SECK ${ }^{1,2}$ and THIERNO SARR ${ }^{3}$ \\ ${ }^{1}$ Département de Mathématiques de la FASTEF ex ENS \\ de I'Université Cheikh Anta Diop \\ de Dakar \\ Sénégal \\ ${ }^{2}$ Laboratoire d'Analyse Numérique et d'Informatique \\ Université Gaston Berger \\ BP 234, Saint-Louis \\ Senegal \\ e-mail: cseckdiakhao@gmail.com \\ cheikh5.seck@ucad.edu.sn \\ ${ }^{3}$ Département de Mathématiques et Informatique \\ de la Faculté des Sciences et Techniques \\ I'Université Cheikh Anta Diop \\ de Dakar \\ Sénégal \\ e-mail: thiernosarr@gmx.fr
}




\begin{abstract}
In this work, we study the exact controllability of a system of two coupled wave equations. We first show that this system has a solution in the space of the usual energy; and using a method adapted multipliers, we found two observability inequalities that allow us to study the existence of a border control that returns the system to a state of equilibrium.
\end{abstract}

\title{
1. Statement of Problem
}

Let $\Omega$ be a non empty bounded domain of $\mathbb{R}^{2}$ of boundary $\Gamma$ regular class $C^{2}$ such that $\Gamma=\Gamma_{e} \cup \bar{\Gamma}_{e}, \Gamma_{e} \cap \bar{\Gamma}_{e}=\emptyset, \omega_{1} \subset \Omega$ and $\omega_{2} \subset \Omega$ two subset such that $\omega_{1} \cap \omega_{2} \neq \emptyset$.

Consider the following coupled system:

$$
\left\{\begin{array}{c}
\phi^{\prime \prime}-\Delta \phi+\alpha y_{t}=g(t) \chi_{\omega_{1}} \text { in } \Omega \times \mathbb{R}^{+}, \\
y^{\prime \prime}-\Delta y-\alpha \phi_{t}=g(t) \chi_{\omega_{2}} \text { in } \Omega \times \mathbb{R}^{+}, \\
\phi=0 \text { on } \Gamma \times \mathbb{R}^{+}, \\
y=0 \text { on } \Gamma_{e} \times \mathbb{R}^{+}, \\
y=v \text { on } \bar{\Gamma}_{e} \times \mathbb{R}^{+}, \\
(\text {i.c. })\left\{\begin{array}{c}
(\phi(x, 0), y(x, 0))=\left(\phi_{0}, y_{0}\right), \\
\left(\phi_{t}(x, 0), y_{t}(x, 0)\right)=\left(\phi_{1}, y_{1}\right),
\end{array}\right.
\end{array}\right.
$$

with $0<\alpha \leq 1$ and $f, g$ two regular functions in $\Omega$, in particular respectively on $\omega_{1}$ and $\omega_{2}$.

Many authors are already investigated this kind of problem (1.1).

In [6], Khodja and Bader studied indirect observability of a system of wave equations weakly coupled by multiplier method, the author has shown that, for a time $T>0$ large enough, the observation of the normal derivative on the edge $\bar{\Gamma}_{e}$ can reproduce a weakened energy of the initial 
data. Also, in [12], Liu and Rao studied indirect exact controllability for one-dimensional system of two weakly coupled wave equations; and, a nonharmonic analysis, they established inequalities observability low and showed the exact controllability for regular initial data. In [7], Khodja et al. studied the problems of stability of a system of equations of twodimensional waves under the effect of a single internal control. In this paper, they showed that the internal control which acts on only one of the equations do not give the exponential stability if the propagation speeds are different. Also, Wehbe [1], Liu \& Rao [12] and Khodja et al. [7] studied respectively the indirect internal observability and stabilization of a linear system of two equations of the waves.

Our modest contribution is to find two observability inequalities that have allowed us to study the existence of a boundary control that allows the precise controllability of the system in a smooth domain.

\section{Observability Inequalities}

\subsection{Study of homogeneous system}

Consider the following homogeneous system:

$$
\left\{\begin{array}{c}
\phi^{\prime \prime}-\Delta \phi+\alpha y_{t}=0 \text { in } \Omega \times \mathbb{R}^{+}, \\
y^{\prime \prime}-\Delta y-\alpha \phi_{t}=0 \text { in } \Omega \times \mathbb{R}^{+}, \\
\phi=0 \text { on } \Gamma \times \mathbb{R}^{+}, \\
y=0 \text { on } \Gamma_{e} \times \mathbb{R}^{+},
\end{array}\right.
$$

with the following initial conditions:

$$
(\text { i.c. })\left\{\begin{array}{c}
\phi(x, 0)=\phi_{0}(x) \phi_{t}(x, 0)=\phi_{1}(x), \\
y(x, 0)=y_{0}(x) y_{t}(x, 0)=y_{1}(x), \forall x \in \Omega .
\end{array}\right.
$$


Let $(\phi, y)$ be a regular solution of system (2.1). We define the associated energy of the system (2.1) by

$$
E(t)=\frac{1}{2} \int_{\Omega}\left(\left|\phi_{t}\right|^{2}+|\nabla \phi|^{2}+\left|y_{t}\right|^{2}+|\nabla y|^{2}\right) d x .
$$

Let $\mathcal{H}=\left(H_{0}^{1}(\Omega) \times L^{2}(\Omega)\right)^{2}$ be the space of the energy of system $(2.1) ; \mathcal{H}$ is a Hilbert space.

We equip $\mathcal{H}$ with the following scalar product:

$\forall \Phi, \bar{\Phi} \in \mathcal{H}$, the following bracket is defined by:

$$
\langle\Phi, \bar{\phi}\rangle_{\mathcal{H}}=\int_{\Omega}(\nabla \phi . \nabla \widetilde{\phi}+\zeta \bar{\zeta}+\nabla y \cdot \nabla \bar{y}+\eta \bar{\eta}) d x
$$

with $\Phi=(\phi, \zeta, y, \eta), \bar{\Phi}=(\bar{\phi}, \bar{\zeta}, \bar{y}, \bar{\eta}) \in \mathcal{H}$. Therefore, we define unbounded linear operator $\mathcal{A}$ by:

$$
D(\mathcal{A})=\left\{\Phi=(\phi, \zeta, y, \eta) \in \mathcal{H} ; \phi, y \in H^{2}(\Omega) \cap H_{0}^{1}(\Omega) ; \zeta, \eta \in H_{0}^{1}(\Omega)\right\},
$$

and

$$
\mathcal{A} \Phi=(\zeta, \Delta \phi-\alpha \eta, \eta, \Delta y+\alpha \zeta), \forall \Phi=(\phi, \zeta, y, \eta) \in D(\mathcal{A})
$$

Thus, an abstract formulation of the system (2.1) gives:

$$
\left\{\begin{array}{c}
\Phi_{t}=\mathcal{A} \Phi \\
\Phi(0)=\Phi_{0} \in \mathcal{H} .
\end{array}\right.
$$

According to Wehbe [1], the operator $\mathcal{A}$ defined is $m$-dissipative in the space of energy $\mathcal{H}$. 


\subsection{Existence and uniqueness result}

Proposition 2.1 (Existence and uniqueness).

(1) Let $\Phi_{0}=\left(\phi_{0}, \phi_{1}, y_{0}, y_{1}\right) \in D(\mathcal{A})$. The system (2.7) admit a solution $\Phi(t)$ that verify $\Phi(t) \in \mathcal{C}^{0}\left(\left[0, \infty[; D(\mathcal{A})) \cap \mathcal{C}^{1}([0, \infty[; \mathcal{H})\right.\right.$.

(2) Let $\Phi_{0}=\left(\phi_{0}, \phi_{1}, y_{0}, y_{1}\right) \in \mathcal{H}$. The system (2.7) admit a weak solution $\Phi(t)$ that satisfy $\Phi(t) \in \mathcal{C}^{0}([0, \infty[; \mathcal{H})$.

(3) Moreover, we have

$$
\left\|\Phi_{0}\right\|_{\mathcal{H}}^{2}=\|\Phi(t)\|_{\mathcal{H}}^{2}, \quad \forall t \in \mathbb{R}^{+}
$$

Proof. (1) Also, either $\Phi_{0} \in D(\mathcal{A})$. according to Hille-Yosida theorem (see Brezis [3]), the problem (2.1) has a solution $\Phi=\left(\phi, \phi_{t}, y, y_{t}\right)$ $\in \mathcal{C}^{0}\left(\mathbb{R}^{+}, D(\mathcal{A}) \cap \mathcal{C}^{1}\left(\mathbb{R}^{+}, \mathcal{H}\right)\right)$.

(2) Let $\Phi_{0} \in \mathcal{H}$. Then, according to Hille-Yosida theorem, the problem (2.1) admit a single weak solution $\Phi=\left(\phi, \phi_{t}, y, y_{t}\right)$, such that

(i) $(\phi(t), y(t)) \in \mathcal{C}^{0}\left(\mathbb{R}^{+}, H_{0}^{1}(\Omega)\right) \cap \mathcal{C}^{1}\left(\mathbb{R}^{+}, \mathcal{H}\right)$,

(ii) $\left(\phi_{t}(t), y_{t}(t)\right) \in \mathcal{C}^{0}\left(\mathbb{R}^{+}, L^{2}(\Omega)\right)$.

\subsection{Reminder results of observability}

In the domain $\Omega \subset R^{2}$, assume the following geometric conditions:

For every $r>0, m(x)=x-x_{0}$ and (,) denotes the scalar product on the space such that we have there geometrically:

$$
\text { (g.c.) }\left\{\begin{array}{l}
m . \nu \geq r^{-1}, \forall x \in \bar{\Gamma}_{e}, \\
m . \nu \leq 0, \forall x \in \Gamma_{e} .
\end{array}\right.
$$

According to Wehbe [1] and Rao [12], we have the result of indirect boundary observability following: 
Lemma 2.2 (Wehbe-Rao). We assume that $0<\beta<\beta_{0}=(4 R+3$. $\left.\max \left\{1, C_{p}\right\}\right)^{-1}$, where $C_{p}$ is the constant of Poincarre and $R=\max _{x \in \Omega}$ $\left\|x-x_{0}\right\|$. There exists a constant $T_{0}>0$ such that for any $T>T_{0}$ and all $\Phi \in \mathcal{H}$, the weak solution $\Phi(x, t)=\left(\phi, \phi_{t}, \psi, \psi_{t}\right)$ to the system (3.1) verifies

$$
C_{2} \int_{0}^{T} \int_{\bar{\Gamma}_{e}}\left|\frac{\partial \psi}{\partial \nu}\right|^{2} d \sigma d t \leq E_{0} \leq C_{3} \int_{0}^{T} \int_{\bar{\Gamma}_{e}}\left|\frac{\partial \psi}{\partial \nu}\right|^{2} d \sigma d t
$$

where $C_{2}, C_{3}$ are positif constants and $T_{0}=\frac{\frac{6}{\beta}+8 R+6 \max \left\{1, C_{0}\right\}}{1-\beta\left(4 R+3 \max \left\{1, C_{0}\right\}\right)}$.

For proof of this lemma, see Liu-Rao [12].

\section{Main Results of Indirect Exact Controllability}

Consider again the following system with the above conditions

$$
\left\{\begin{array}{c}
\phi^{\prime \prime}-\Delta \phi+\alpha y_{t}=f(t) \chi_{\omega_{1}} \text { in } \Omega \times \mathbb{R}^{+}, \\
y^{\prime \prime}-\Delta y-\alpha \phi_{t}=g(t) \chi_{\omega_{2}} \text { in } \Omega \times \mathbb{R}^{+}, \\
\phi=0 \text { on } \Gamma \times \mathbb{R}^{+}, \\
y=0 \text { on } \Gamma_{e} \times \mathbb{R}^{+}, \\
y=v \text { on } \bar{\Gamma} \times \mathbb{R}^{+}, \\
(i . c .)\left\{\begin{array}{l}
(\phi(x, 0), y(x, 0))=\left(\phi_{0}, y_{0}\right), \\
\left(\phi_{t}(x, 0), y_{t}(x, 0)\right)=\left(\phi_{1}, y_{1}\right) .
\end{array}\right.
\end{array}\right.
$$

The system (3.1) can be reformulated and solved by the method of transposition (see Lions [10, 11] or Komornik [13]).

It arises, so of course, the problem of indirect (or implicit) exact controllability as follows:

Given $T>0$ and suitable initial data $\Phi_{0}=\left(\phi_{0}, \phi_{1}, y_{0}, y_{1}\right)$, is there a control $v$ which reduces the solution (3.1) from time to time in the equilibrium state $T$, i.e., $\phi(T)=\phi_{t}(T)=y(T)=y_{t}(T)=0$ ? 
Theorem 3.1 (Main result). Let $T>T_{0}>0$ ( $T$ large enough, $T_{0}$ minimal observability time) and $v \in L^{2}\left([0, T], L^{2}\left(\bar{\Gamma}_{e}\right)\right), f \in L^{2}\left(\omega_{1} \times[0, T]\right)$ and $g \in L^{2}\left(\omega_{2} \times[0, T]\right)$.

For any initial data $\Phi_{0}=\left(\phi_{0}, \phi_{1}, y_{0}, y_{1}\right) \in\left(L^{2}(\Omega) \times H^{-1}(\Omega)\right)^{2}$, we have

(i) The system (3.1) admit a weak solution

$$
\Phi(x, t) \in \mathcal{C}^{0}\left([0, T],\left(L^{2}(\Omega) \times H^{-1}(\Omega)\right)^{2}\right) .
$$

(ii) Moreover, for all $\Phi_{0} \in\left(L^{2}(\Omega) \times H^{-1}(\Omega)\right)^{2}$ and $v \in L^{2}\left([0, T] ; L^{2}\left(\Gamma_{e}\right)\right)$, the bilinear form $a:\left(\Phi_{0}, v\right) \mapsto\left(\Phi, \Phi_{t}\right)$ is continuous from

$$
\left(L^{2}(\Omega) \times H^{-1}(\Omega)\right)^{2} \times L^{2}\left([0, T] ; L^{2}\left(\bar{\Gamma}_{e}\right)\right) \text { to } \mathcal{C}^{0}\left([0, T] ;\left(L^{2}(\Omega) \times H^{-1}(\Omega)\right)^{2}\right)
$$

Proof of main Theorem 3.1. Let $\Phi=\left(\phi, \phi_{t}, \psi_{0}, \psi_{1}\right)$.

Multiplying the first equation of (3.1) by $\varphi$ and the second by $\psi$ and integrating by part (Green) we obtain:

$$
\begin{aligned}
& \int_{\Omega} \phi_{t}(t) \varphi(t) d x+\int_{\Omega} y_{t}(t) \psi(t) d x-\int_{\Omega} \phi(t) \varphi(t) d x-\int_{\Omega} y(t) \psi_{t}(t) d x \\
& -\int_{\Omega} \alpha \varphi(t) \psi(t) d x-\int_{\Omega} \alpha y(t) \varphi(t) d x+\int_{\Omega} f(t) \chi_{\omega 1} \varphi(t) d x+\int_{\Omega} g(t) \chi_{\omega 2} \psi(t) d x \\
& =\int_{\Omega} \phi_{t}(0) \varphi(0) d x+\int_{\Omega} y_{t}(0) \psi(0) d x-\int_{\Omega} \phi(0) \varphi(0) d x-\int_{\Omega} y(0) \psi_{t}(0) d x \\
& -\int_{\Omega} \alpha \varphi(0) \psi(0) d x-\int_{\Omega} \alpha y(0) \varphi(0) d x+\int_{\omega_{1}} f(t) \varphi(t) d x+\int_{\omega_{2}} g(t) \psi(t) d x \\
& -\int_{\bar{\Gamma}_{e}} \int_{0}^{T} \frac{\partial \psi}{\partial \nu} v d \sigma d t
\end{aligned}
$$

Note by $\mathcal{H}^{\prime}=H^{-1}(\Omega) \times L^{2}(\Omega)$ the dual of the space $\mathcal{H}$. 
Let $l$ be the linear form defined from the relationship (3.4) by:

$$
\begin{aligned}
l\left(\Phi_{0}\right)= & \left\langle\left(\phi_{t},-\phi, y_{t},-y\right), \Phi(t)\right\rangle_{\mathcal{H}^{\prime} \times \mathcal{H}} \\
= & \left\langle\left(\phi_{1},-\phi_{0}, y_{1},-y_{0}\right), \Phi(0)\right\rangle_{\mathcal{H} \times \mathcal{H}}-\int_{\Gamma} \int_{0}^{t} \frac{\partial \psi}{\partial \nu} d \sigma d t \\
= & \int_{\Omega} \phi_{t}(0) \varphi(0) d x+\int_{\Omega} y_{t}(0) \psi(0) d x \\
& -\int_{\Omega} \phi(0) \varphi(0) d x-\int_{\Omega} y(0) \varphi_{t}(0) d x-\int_{\Omega} \alpha \varphi(0) \psi(0) d x \\
& -\int_{\Omega} \alpha y(0) \varphi(0) d x+\int_{\omega_{1}} f(0) \varphi(0) d x+\int_{\omega_{2}} g(0) \psi(0) d x
\end{aligned}
$$

for all $\Phi_{0} \in \mathcal{H}$.

For all control $v \in L^{2}\left(0, T, L^{2}\left(\bar{\Gamma}_{e}\right)\right)$, we have

$$
|l(\Phi)| \leq C(\Omega)\left(\left\|U_{0}\right\|_{\mathcal{H}^{\prime}}+\|v\|_{L^{2}\left(0, T ; L^{2}\left(\bar{\Gamma}_{e}\right)\right)}\right)\left\|\Phi_{0}\right\|_{\mathcal{H}},
$$

where $U_{0}=\left(u_{0}, v_{0}, \eta_{0}\right) \in\left(L^{2}(\Omega) \times H^{-1}(\Omega)\right)^{2}=\mathcal{H}$ and $\Phi_{0}$ the initial conditions.

So $l$ is continuous in $\mathcal{H}$.

Moreover, we have

$$
\|l\|_{L^{2}(\mathcal{H}, \mathbb{R})} \leq\left\|U_{0}\right\|_{\mathcal{H}^{\prime}}+\|v\|_{L^{2}\left(0, T ; L^{2}\left(\bar{\Gamma}_{e}\right)\right)}
$$

From Riesz theorem, there exists a function $Z(x, t) \in \mathcal{H}^{\prime}$ as

$$
l\left(\Phi_{0}\right)=\left\langle Z, \Phi_{0}\right\rangle_{\mathcal{H}^{\prime} \times \mathcal{H}}, \forall \Phi_{0} \in \mathcal{H}
$$

Let us define the solution $U(x, t)$ by: 
$S_{\mathcal{A}}^{*}(t) U(x, t)=Z(x, t) ; U(x, t)$ thus defined is the unique solution of the system (3.2) for all $0 \leq t \leq T$ and $S_{\mathcal{A}}^{*}(t)$ the dual of semi-group of contractions $S_{\mathcal{A}}(t)$ associated of system (2.7).

Moreover, we have:

$$
\begin{aligned}
\|U(x, t)\|_{\mathcal{H}^{\prime}} & =\left\|l\left(v, U_{0} ; t\right)\right\|_{L^{2}(\mathcal{H}, \mathbb{R})} \\
& \left.\leq C(\Omega)\|v\|_{L^{2}\left(0, T ; L^{2}\left(\bar{\Gamma}_{e}\right)\right)}+\left\|U_{0}\right\|_{\mathcal{H}^{\prime}}\right), \forall t \in[0, T] .
\end{aligned}
$$

Hence the linear form $l$ is continuous from $\left(L^{2}(\Omega) \times H^{-1}(\Omega)\right)^{2} \times\left(L^{2}\right.$ $\left([0, T] ; L^{2}\left(\bar{\Gamma}_{e}\right)\right)$ to $\mathcal{C}^{0}\left(0, T ;\left(L^{2}(\Omega) \times H^{-1}(\Omega)\right)^{2}\right)$.

\subsection{Exact controllability}

Theorem 3.2 (Exact controllability). Assume that $0<\beta<\beta_{0}$, for all $T>T_{0}$ and $U_{0} \in\left(L^{2}(\Omega) \times H^{-1}(\Omega)\right)^{2}$. There is a dynamic border control $v(t) \in L^{2}\left([0, T] ; L^{2}\left(\bar{\Gamma}_{e}\right)\right)$ such that the solution of system (3.1) verify $\phi(T)=\phi_{t}(T)=y(T)=y_{t}(T)=0, \quad$ i.e., the system (3.1) is exactly controllable.

Proof. Let $\Phi=\left(\phi, \phi_{t}, \psi, \psi_{t}\right) \in \mathcal{H}$. And, for $\Phi_{0} \in \mathcal{H}$, we define the semi-norm

$$
\left\|\Phi_{0}\right\|_{\mathcal{H}}^{2}=\int_{0}^{T} \int_{\bar{\Gamma}_{e}}\left|\frac{\partial \psi}{\partial \nu}\right|^{2} d \sigma d t
$$

$\Phi$ designating the homogeneous solution of the problem (3.1). Choose the border control $v=-\frac{\partial \psi}{\partial \nu} \in L^{2}\left(0, T, L^{2}\left(\bar{\Gamma}_{e}\right)\right)$. 
Consider the following retrograde problem:

$$
\left\{\begin{array}{c}
\varepsilon^{\prime \prime}-\Delta \varepsilon+\alpha \zeta_{t}=f(t) t \chi_{\omega_{1}} \text { in } \Omega \times \mathbb{R}^{+}, \\
\zeta^{\prime \prime}-\Delta \zeta-\alpha \varepsilon_{t}=g(t) \chi_{\omega_{2}} \text { in } \Omega \times \mathbb{R}^{+}, \\
\varepsilon=0 \text { on } \Gamma \times \mathbb{R}^{+}, \\
\zeta=0 \text { on } \Gamma_{e} \times \mathbb{R}^{+}, \\
\zeta=-\frac{\partial \psi}{\partial \nu} \text { on } \bar{\Gamma}_{e} \times \mathbb{R}^{+}, \\
(f . c .)\left\{\varepsilon(T)=\varepsilon_{t}(T)=\zeta(T)=\zeta_{t}(T)=0 \text { on } \Omega .\right.
\end{array}\right.
$$

We know that (see Lions [11], Sz-Nagy and Foias [2]) the system (3.10) admit a unique solution $\Psi(x, t)=\left(\varepsilon, \varepsilon_{t}^{f}, \zeta, \zeta_{t}^{g}\right) \in \mathcal{C}^{0}\left([0, T] ; \mathcal{H}^{\prime}\right)$.

Let us define the linear operator $\Lambda$ by:

$\Lambda: \mathcal{H} \rightarrow \mathcal{H}^{\prime}=\left(H^{-1}(\Omega) \times L^{2}(\Omega)\right)^{2}$ where $\Lambda \Phi_{0}=\left(\psi_{t}(0),-\psi(0), \zeta_{t}(0)\right.$, $-\zeta(0)), \forall \Phi_{0} \in \mathcal{H}$.

Moreover, we have

$$
\left\{\begin{aligned}
\left\langle\Lambda \Phi_{0}, \widetilde{\Phi}_{0}\right\rangle_{\mathcal{H}^{\prime} \times \mathcal{H}} & =\int_{O}^{T} \int_{\bar{\Gamma}_{e}}\left(\frac{\partial \psi}{\partial \nu} \frac{\partial \widetilde{\varphi}}{\partial \nu}\right) d \sigma d t \\
& =\left(\Phi_{0}, \widetilde{\Phi}_{0}\right)_{\mathcal{H}^{\prime}}
\end{aligned}\right.
$$

The Cauchy-Schwartz inequality applied to the relationship (3.11) give

$$
\left|\left\langle\Lambda \Phi_{0}, \widetilde{\Phi}_{0}\right\rangle_{\mathcal{H}^{\prime} \times \mathcal{H}}\right| \leq\left\|\Phi_{0}\right\|_{\mathcal{H}}\left\|\widetilde{\Phi}_{0}\right\|_{\mathcal{H}}, \quad \forall \Phi_{0}, \widetilde{\Phi}_{0} \in \mathcal{H}
$$

In particular, we have

$$
\left|\left\langle\Lambda \Phi_{0}, \Phi_{0}\right\rangle\right| \leq\left\|\Phi_{0}\right\|_{\mathcal{H}}^{2}, \quad \forall \Phi_{0} \in \mathcal{H}
$$

From theorem of the inverse inequality observabilty (see Lions [10, 11]), we know that the operator $\Lambda$ is linear, continuous and coercive on $\mathcal{H}$. So, according to the Lax-Milgram theorem, $\Lambda$ is an isomorphism of $\mathcal{H}$ in $\mathcal{H}^{\prime}$. 
Therefore, for any $U_{0} \in\left(L^{2}(\Omega) \times H^{-1}(\Omega)\right)^{2}$, there exists a unique solution $\Phi_{0} \in \mathcal{H}$, such that

$$
\Lambda \Phi_{0}=\left(\phi_{1},-\phi_{0}, y_{1},-y_{0}\right)
$$

A uniqueness theorem of the solution leads to that $U=\Psi$. From where we have the result of exact controllability following: $\phi(T)=\phi_{t}(T)=y(T)$ $=y_{t}(T)=0$.

\section{References}

[1] A. Wehbe, Rational energy decay rate for a wave equation with dynamical control, Applied Mathematics Letters 16(3) (2003), 357-364.

DOI: https://doi.org/10.1016/S0893-9659(03)80057-5

[2] B. Sz.-Nagy and C. Foias, Analyse Harmonique d'Opérateurs de l'Espace de Hilbert, Masson, Paris, 1967.

[3] Haïm Brezis, Analyse fonctionnelle, Collection Mathématiques Appliquées pour la Maîtrise, Théorie et Applications, Masson, Paris, 1983, xiv + 234, 2 - 225 - 77198 $7,46-01(47-01), 697382(85 a$ : 46001).

[4] C. D. Benchimol, A note on weak stabilizability of contraction semigroups, SIAM Journal on Control and Optimization 16(3) (1978), 373-379.

DOI: https://doi.org/10.1137/0316023

[5] C. Seck, A. Sène and M. T. Niane, Estimates related to the extended spectral control of the wave equation, Journal of Mathematics Research 10(4) (2018), 156-164.

DOI: https://doi.org/10.5539/jmr.v10n4p156

[6] Farid Ammar Khodja and Ahmed Bader, Stabilizability of systems of onedimensional wave equations by one internal or boundary control force, SIAM Journal on Control and Optimization 39(6) (2001), 1833-1851.

DOI: https://doi.org/10.1137/S0363012900366613

[7] Farid Ammar Khodja, Assia Benabdallah and Djamel Teniou, Stability of coupled systems, Abstract and Applied Analysis 1(3) (1996); Article ID 901468, pages 14.

DOI: https://doi.org/10.1155/S1085337596000176

[8] F. Huang, Strong asymptotic stability of linear dynamical systems in Banach spaces, Journal of Differential Equations 104(2) (1993), 307-324.

DOI: https://doi.org/10.1006/jdeq.1993.1074 
[10] J.-L. Lions, Contrôlabilité exacte, perturbations et stabilisation de systemes distributes: Tome 1, Recherches en Mathématiques Appliquées, Research in Applied Mathematics, Volume 8, Paris, 1988.

[11] J.-L. Lions, Contrôlabilité exacte, perturbations et stabilisation de systèmes distributes: Tome 2, Recherches en Mathématiques Appliquées, Research in Applied Mathematics.

[12] B. Rao and Z. Liu, A spectral approach to the indirect boundary control of a system of weakly coupled wave equations, Discrete and Continuous Dynamical Systems: A 23(1-2) (2009), 399-414.

DOI: https://doi.org/10.3934/dcds.2009.23.399

[13] V. Komornik, Exact Controllability and Stabilization: The Multiplier Method, Collection R. M. A. Éditions Masson et J. Wiley, 1994. 